\title{
Dietary Advanced Glycation End-Products and Colorectal Cancer Risk in the European Prospective Investigation into Cancer and Nutrition (EPIC) Study
}

\author{
Elom K. Aglago ${ }^{1}$, Ana-Lucia Mayén ${ }^{1}$, Viktoria Knaze ${ }^{2}$, Heinz Freisling ${ }^{1}$ D, Veronika Fedirko ${ }^{3}$, \\ David J. Hughes ${ }^{4} \mathbb{D}$, Li Jiao ${ }^{5} \mathbb{D}$, Anne Kirstine Eriksen ${ }^{6}$, Anne Tjønneland ${ }^{6} \mathbb{D}$, Marie-Christine Boutron-Ruault ${ }^{7,8}$, \\ Joseph A. Rothwell 7,8, Gianluca Severi ${ }^{7,8,9}$, Rudolf Kaaks ${ }^{10}$, Verena Katzke 10, Matthias B. Schulze 11,12 ${ }^{10}$, \\ Anna Birukov ${ }^{11}\left(\mathbb{D}\right.$, Domenico Palli ${ }^{13}(\mathbb{D})$, Sabina Sieri ${ }^{14}{ }^{(D)}$, Maria Santucci de Magistris ${ }^{15}$, Rosario Tumino ${ }^{16}$, \\ Fulvio Ricceri ${ }^{17,18}$, Bas Bueno-de-Mesquita ${ }^{19}$, Jeroen W. G. Derksen ${ }^{20}$, Guri Skeie ${ }^{21}$ (D), Inger Torhild Gram ${ }^{21}(\mathbb{D}$, \\ Torkjel Sandanger ${ }^{21}$, J. Ramón Quirós ${ }^{22}$, Leila Luján-Barroso ${ }^{23}$ D, Maria-Jose Sánchez 24,25,26,27, Pilar Amiano 24,28, \\ María-Dolores Chirlaque ${ }^{24,29}$, Aurelio Barricarte Gurrea 24,30,31, Ingegerd Johansson ${ }^{32}{ }^{\mathbb{D}}$, Jonas Manjer ${ }^{33,34}$, \\ Aurora Perez-Cornago ${ }^{35}$, Elisabete Weiderpass ${ }^{36}$ (D), Marc J. Gunter ${ }^{1}$, Alicia K. Heath ${ }^{37}$ (D), \\ Casper G. Schalkwijk ${ }^{38,+}$ and Mazda Jenab ${ }^{1, *,+}$
}

Citation: Aglago, E.K.; Mayén, A.-L. Knaze, V.; Freisling, H.; Fedirko, V.; Hughes, D.J.; Jiao, L.; Eriksen, A.K.; Tjønneland, A.; Boutron-Ruault, M.-C.; et al. Dietary Advanced Glycation End-Products and Colorectal Cancer Risk in the European Prospective Investigation into Cancer and Nutrition (EPIC) Study. Nutrients 2021, 13, 3132. https://doi.org/10.3390/nu13093132

Academic Editor: Rachel Murphy

Received: 10 August 2021

Accepted: 2 September 2021

Published: 8 September 2021

Publisher's Note: MDPI stays neutral with regard to jurisdictional claims in published maps and institutional affiliations.

Copyright: (c) 2021 by the authors Licensee MDPI, Basel, Switzerland. This article is an open access article distributed under the terms and conditions of the Creative Commons Attribution (CC BY) license (https:// creativecommons.org/licenses/by/ $4.0 /)$.
1 Nutrition and Metabolism Section, International Agency for Research on Cancer (IARC), 69372 Lyon, France; aglagoe@fellows.iarc.fr (E.K.A.); mayenchacona@fellows.iarc.fr (A.-L.M.); freislingh@iarc.fr (H.F.); GunterM@iarc.fr (M.J.G.)

2 Early Detection, Prevention, and Infections Branch, International Agency for Research on Cancer (IARC), 69372 Lyon, France; knazev@iarc.fr

3 Department of Epidemiology, The University of Texas MD Anderson Cancer Center, Houston, TX 77030, USA; VFedirko@mdanderson.org

4 Cancer Biology and Therapeutics Group (CBT), Conway Institute, School of Biomolecular and Biomedical Science (SBBS), University College Dublin, D04 V1W8 Dublin, Ireland; david.hughes@ucd.ie

5 Department of Medicine, Baylor College of Medicine, Houston, TX 77030, USA; jiao@bcm.edu

6 Danish Cancer Society Research Center, 2100 Copenhagen, Denmark; ake@cancer.dk (A.K.E.); annet@cancer.dk (A.T.)

7 CESP, Faculté de Médecine-Université Paris-Saclay, UVSQ, INSERM, 94805 Villejuif, France; Marie-christine.BOUTRON@gustaveroussy.fr (M.-C.B.-R.); Joseph.ROTHWELL@gustaveroussy.fr (J.A.R.); Gianluca.SEVERI@gustaveroussy.fr (G.S.)

8 Gustave Roussy, 114, Rue Édouard-Vaillant, CEDEX, 94805 Villejuif, France

9 Department of Statistics, Computer Science and Applications (DISIA), University of Florence, 50121 Florence, Italy

10 Division of Cancer Epidemiology, German Cancer Research Center (DKFZ), 69120 Heidelberg, Germany; r.kaaks@Dkfz-Heidelberg.de (R.K.); V.Katzke@Dkfz-Heidelberg.de (V.K.)

11 Department of Molecular Epidemiology, German Institute of Human Nutrition Potsdam-Rehbruecke, 14558 Nuthetal, Germany; mschulze@dife.de (M.B.S.); Anna.Birukov@dife.de (A.B.)

12 Institute of Nutrition Science, University of Potsdam, 14558 Nuthetal, Germany

13 Cancer Risk Factors and Life-Style Epidemiology Unit, Institute for Cancer Research, Prevention and Clinical Network (ISPRO), 50139 Florence, Italy; d.palli@ispro.toscana.it

14 Epidemiology and Prevention Unit, Fondazione IRCCS Istituto Nazionale dei Tumori di Milano, 20133 Milan, Italy; Sabina.Sieri@istitutotumori.mi.it

15 Azienda Ospedaliera Universitaria Federico II, 80131 Napoli, Italy; masantuc@unina.it

16 Hyblean Association for Epidemiological Research AIRE-ONLUS, 97100 Ragusa, Italy; rtuminomail@gmail.com

17 Department of Clinical and Biological Sciences, University of Turin, Regione Gonzole 10, 10043 Orbassano, Italy; fulvio.ricceri@unito.it

18 Unit of Epidemiology, Regional Health Service ASL TO3, Via Sabaudia 164, 10095 Grugliasco, Italy

19 Department for Determinants of Chronic Diseases (DCD), National Institute for Public Health and the Environment (RIVM), P.O. Box 1, 3720 BA Bilthoven, The Netherlands; basbuenodemesquita@gmail.com

20 Julius Center for Health Sciences and Primary Care, University Medical Center Utrecht, Utrecht University, 3584 CG Utrecht, The Netherlands; H.W.G.Derksen-2@umcutrecht.nl

21 Faculty of Health Sciences, Department of Community Medicine, University of Tromsø, The Arctic University of Norway, 9037 Tromsø, Norway; guri.skeie@uit.no (G.S.); inger.gram@uit.no (I.T.G.); torkjel.sandanger@uit.no (T.S.)

22 Public Health Directorate, 33402 Asturias, Spain; JOSERAMON.QUIROSGARCIA@asturias.org 
23 Unit of Nutrition and Cancer, Catalan Institute of Oncology-ICO; and Nutrition and Cancer Group; Epidemiology, Public Health, Cancer Prevention and Palliative Care Program, Bellvitge Biomedical Research Institute—IDIBELL, L'Hospitalet de Llobregat, Av. Granvia 199-203, 08908 Barcelona, Spain; llujan@iconcologia.net

24 Centro de Investigación Biomédica en Red de Epidemiología y Salud Pública (CIBERESP), 28029 Madrid, Spain; mariella_san@hotmail.com (M.-J.S.); epicss-san@euskadi.eus (P.A.); mdolores.chirlaque@carm.es (M.-D.C.); aurelio.barricarte.gurrea@navarra.es (A.B.G.) Escuela Andaluza de Salud Pública (EASP), 18011 Granada, Spain

26 Instituto de Investigación Biosanitaria ibs.GRANADA, 18012 Granada, Spain

27 Department of Preventive Medicine and Public Health, University of Granada, 18071 Granada, Spain

28 Public Health Division of Gipuzkoa, BioDonostia Research Institute, 20014 Donostia-San Sebastian, Spain

29 Department of Epidemiology, Murcia Regional Health Council, IMIB-Arrixaca, Murcia University, 30003 Murcia, Spain

30 Navarra Public Health Institute, 31008 Pamplona, Spain

31 Navarra Institute for Health Research (IdiSNA), 31008 Pamplona, Spain

32 Department of Radiation Sciences, Oncology, Umeå University, 90736 Umeå, Sweden; ingegerd.johansson@umu.se

33 Department of Clinical Sciences, Malmö, Lund University, 22100 Lund, Sweden; jonas.manjer@med.lu.se

34 Division of Surgery, Malmö, Lund University, 22100 Lund, Sweden

35 Cancer Epidemiology Unit, Nuffield Department of Population Health, University of Oxford, Oxford OX3 7LF, UK; Aurora.Perez-Cornago@ndph.ox.ac.uk

36 Office of the Director, International Agency for Research on Cancer (IARC), 69372 Lyon, France; WeiderpassE@iarc.fr

37 Department of Epidemiology and Biostatistics, School of Public Health, Imperial College London, London W2 1PG, UK; a.heath@imperial.ac.uk

38 Department of Internal Medicine, CARIM School for Cardiovascular Diseases, Maastricht University Medical Center, 6229 HX Maastrich, The Netherlands; c.schalkwijk@maastrichtuniversity.nl

* Correspondence: jenabm@iarc.fr; Tel.: +33-472-73-80-82

+ These authors contributed equally to this work.

\begin{abstract}
Dietary advanced glycation end-products (dAGEs) have been hypothesized to be associated with a higher risk of colorectal cancer (CRC) by promoting inflammation, metabolic dysfunction, and oxidative stress in the colonic epithelium. However, evidence from prospective cohort studies is scarce and inconclusive. We evaluated CRC risk associated with the intake of dAGEs in the European Prospective Investigation into Cancer and Nutrition (EPIC) study. Dietary intakes of three major dAGEs: $\mathrm{N}^{\varepsilon}$-carboxy-methyllysine (CML), $\mathrm{N}^{\varepsilon}$-carboxyethyllysine (CEL), and $\mathrm{N}^{\delta}$-(5-hydro-5-methyl-4-imidazolon-2-yl)-ornithine (MG-H1) were estimated in 450,111 participants (median follow-up $=13$ years, with 6162 CRC cases) by matching to a detailed published European food composition database. Hazard ratios (HRs) and 95\% confidence intervals (CIs) for the associations of dAGEs with CRC were computed using multivariable-adjusted Cox regression models. Inverse CRC risk associations were observed for CML (HR comparing extreme quintiles: $\left.\mathrm{HR}_{\mathrm{Q} 5 \mathrm{vs} \cdot \mathrm{Q} 1}=0.92,95 \% \mathrm{CI}=0.85-1.00\right)$ and $\mathrm{MG}-\mathrm{H} 1\left(\mathrm{HR}_{\mathrm{Q} 5 \mathrm{vs} \cdot \mathrm{Q} 1}=0.92,95 \% \mathrm{CI}=0.85-1.00\right)$, but not for $\mathrm{CEL}\left(\mathrm{HR}_{\mathrm{Q} 5 \mathrm{vs} \cdot \mathrm{Q} 1}=0.97,95 \% \mathrm{CI}=0.89-1.05\right)$. The associations did not differ by sex or anatomical location of the tumor. Contrary to the initial hypothesis, our findings suggest an inverse association between dAGEs and CRC risk. More research is required to verify these findings and better differentiate the role of dAGEs from that of endogenously produced AGEs and their precursor compounds in CRC development.
\end{abstract}

Keywords: advanced glycation end-products; dietary glycation compounds; colorectal cancer; dietary exposure

\title{
1. Introduction
}

Colorectal cancer (CRC) is the third most common cancer globally, and the second leading cause of cancer-related deaths [1]. The incidence of CRC follows a geographical distribution pattern, with the highest figures observed in Western countries [2], most likely attributable to the "modern" lifestyle and diet rich in energy-dense processed foods 
with poor nutritional value [3-5]. The Western diet is a substantial source of advanced glycation end-products (AGEs), an expansive group of molecules produced by irreversible non-enzymatic combination of reducing sugars and proteins, lipids, or nucleic acids [6]. The typical Western diet can also promote endogenous formation of AGEs by supplying reducing sugars and AGE precursors such as reactive dicarbonyls, i.e., methylglyoxal, glyoxal, glycolaldehyde, and glyceraldehyde [7]. Dietary AGEs (dAGEs) are known for their pro-inflammation and pro-oxidation properties in the colon and have been reported in diverse colonic pathologies, such as inflammatory bowel diseases [8]. Around 70-90\% of AGEs ingested are unabsorbed $[9,10]$ and remain in the gastrointestinal tract where they can interact directly with colon epithelial cells. The human colon is, therefore, potentially exposed to AGEs from the diet, but also from the systemic milieu by way of circulating AGEs [11,12].

AGEs have been hypothesized to be associated with CRC development [13], mostly due to their ability to promote tumor cell growth in vitro [13]. A body of mechanistic evidence has linked AGEs to CRC through stimulation of the pro-inflammatory response via the activation of the receptor of AGEs (RAGE) [14], an increase in colonic barrier permeability-allowing closer interaction of AGEs with colonic epithelium - and consequential leakage of bacterial toxins into the systemic circulation [15]. Notwithstanding these numerous plausible mechanisms, no previous epidemiological studies have investigated the relationship between dAGEs and CRC, probably due to the lack of detailed food composition databases for these compounds. The development of food composition tables for estimating dietary AGEs is recent, and few tables exist, mainly for Japanese foods [16] and more recently for European foods [17]. Due to the large number of different AGEs, the tables developed focused on the major compounds, specifically $\mathrm{N}^{\varepsilon}$-(carboxymethyl)lysine (CML). The European food composition table provides data on CML and, additionally, on two other major dAGEs: $\mathrm{N}^{\varepsilon}$-(carboxyethyl)lysine (CEL) and $\mathrm{N}^{\delta}$-(5-hydro-5-methyl-4imidazolon-2-yl)-ornithine (MG-H1).

Considering the potential direct interaction of dAGEs with the colonic epithelium and their numerous CRC-promoting effects, we hypothesize a positive CRC risk association with higher dAGEs consumption. We evaluated our hypothesis using information on dietary intake of CML, CEL, and MG-H1 in the prospective European Prospective Investigation into Cancer and Nutrition (EPIC) cohort.

\section{Materials and Methods}

\subsection{Study Participants}

We used data from the EPIC study, a large prospective cohort with over half a million participants $(n=521,324)$ from 10 European countries (Denmark, France, Germany, Greece, Italy, the Netherlands, Norway, Spain, Sweden, and the United Kingdom) [18]. In brief, participants aged between 35 and 75 years were recruited from 1992 to 2000 in 23 participating centers. Anthropometric measures, socio-demographic information, and lifestyle and dietary intake data were collected at recruitment from all participants. Standing height, weight, and waist and hip circumferences were measured, with self-reporting exceptions in France, Norway, and Oxford. Body mass index (BMI, in $\mathrm{kg} / \mathrm{m}^{2}$ ) was calculated.

\subsection{Ethical Considerations}

Ethical approval for the EPIC study was obtained from the Ethical Committee of the International Agency for Research on Cancer (IARC) and local ethical committees. All participants provided written consent to participate in the study.

\subsection{Dietary Assessment and dAGEs Estimation}

Usual diet was collected at baseline using a combination of country- or center-specific questionnaires that have been validated to reflect local contexts $[19,20]$. Dietary data were collected during interviews in Greece, Spain, and Naples and Ragusa (Italy), whereas in other EPIC centers self-administrated questionnaires were used. Quantitative dietary 
questionnaires were used in Germany, Greece, the Netherlands, and Northern Italy; semiquantitative food frequency questionnaires were employed in Denmark, Norway, Naples, Umea, and the UK; and in Malmo a combination of a non-quantitative food-frequency questionnaire and a food record was utilized.

To estimate intakes of individual AGEs, we used the database for protein-bound AGEs developed for 190 food items selected from the Dutch cohort of EPIC and the Dutch National Food Consumption survey [17]. These foods were matched to the EPIC food list by name and descriptors, especially considering preparation and processing to expand the EPIC Nutrient Database (ENDB) with extra food components, a procedure used for other nutrients/anti-nutrients and described in detail elsewhere [21,22]. For complex foods with multiple ingredients, the foods were decomposed into specific ingredients or food items to generate EPIC dAGEs composition data for each food item. Thereafter, for each participant, daily intakes of CML, CEL, and MG-H1 were estimated. $\sum$ dAGEs was calculated as the sum of individual dAGEs (CML+CEL+MG-H1) and used to picture overall AGEs intake patterns. The main food contributors of dietary CML and MG-H1 were (from highest to lowest contribution): cereals and cereal products, meats and meat products, cakes, and biscuits (Supplementary Figure S1) [23]. For CEL, the main contributors were meat and meat products, cereal and cereal products, cakes, and biscuits. Dairy products, fish and fish products, and non-alcoholic drinks were also relevant dietary sources of the three dAGEs.

We excluded participants from Greece $(n=26,048)$ due to data use restrictions, those diagnosed with cancer at baseline $(n=25,184)$, those with missing follow-up information $(n=4148)$ or dietary questionnaire data $(n=6259)$, those in the highest or lowest $1 \%$ of energy intake versus energy requirements $(n=9573)$, and a participant who withdrew from EPIC. Our final dataset included 450,111 participants, among whom 318,686 were women $(71 \%)$.

\subsection{Identification of $C R C$ Cases}

Cancer cases were ascertained from cancer registries in Denmark, Italy, the Netherlands, Norway, Spain, Sweden, and the United Kingdom, or by using a combination of sources, including health insurance records, oncology and pathology records, or, in the specific cases of France and Germany, through active follow-up of the participants and their relatives. CRC cases were first incident, and histologically confirmed by a pathologist. We used the International Classification of Diseases for Oncology (ICD-O, codes C18-C20) to define the cases. Colon cancers were defined as tumors that occurred in the cecum, appendix, ascending colon, hepatic flexure, transverse colon, splenic flexure, or the descending or sigmoid colon (C18.0-C18.7), and overlapping and/or unspecified origin tumors (C18.8 and C18.9). Rectal cancers were defined as tumors that occurred at the recto-sigmoid junction (C19) or rectum (C20).

\subsection{Statistical Analyses}

Daily intakes of CML, CEL, and MG-H1 were natural log-transformed and their standardized residuals were computed by regressing the ln-transformed values on participant energy intake and center. Ln-transformed dAGEs were divided into quintiles, with the first quintile used as the reference in all our analyses. Cox proportional hazards regression models stratified by age at recruitment (one-year categories), sex, and center were used to compute hazard ratios (HRs) and 95\% confidence intervals (CIs) for the association between individual dAGEs and CRC risk. Time at entry was age at recruitment, while exit time was set as the age at which any of the following first occurred: CRC diagnosis, death, emigration, or last date at which follow-up was considered complete. To test the trends of the associations, we ran Cox models using median values of each category as a continuous variable. Analyses were also conducted using continuous variables for dAGEs (per $\ln (\mathrm{SD})$ increment). No deviation from the proportional hazards assumption was observed after assessing Schoenfeld residuals. Three main models were run. Model 1 was stratified by age (1-year categories), sex, and center. Model 2 was additionally adjusted for BMI 
(continuous), height (continuous), and lifestyle factors, including education (none; primary; technical and professional; secondary, higher), physical activity (inactive; moderately inactive; moderately active; active), smoking status and intensity (never; current smokers, cigarettes/day: 1-15, 16-25, >26; former smokers who quit: $<=10,11-20,>20$ years; occasional), and total energy intake (kcal/day, continuous). Model 3 was further adjusted for the Mediterranean diet score to consider the diet as a whole, and because this score has also been specifically associated with CRC risk [24]. We considered missing data as a separate category for physical activity $(1.9 \%)$, education $(2.3 \%)$, and smoking $(3.3 \%)$. Restricted cubic splines were used to model possible nonlinear trends $[25,26]$. Linearity of the associations was tested using the likelihood ratio test, comparing the model with only the linear term with the model including both the linear and the cubic spline terms. There was no indication of nonlinear associations in any of our analyses. Analyses (using Model 3) by anatomical subsites of the colorectum were also run for rectal and colon cancer, and, specifically, for proximal and distal colon cancers. Potential differences in the associations by tumor sites, i.e., rectal vs. colon or proximal colon vs. distal colon, were tested using competing risk analyses [27,28]. Stratified analyses by country, BMI, sex, and years of follow-up were conducted and multiplicative interactions were included in the model to evaluate potential heterogeneity. To model the possible impact of reverse causation, we ran sensitivity analyses by excluding the first 2 years of follow-up.

All the analyses were carried out using Stata 14.0 (StataCorp., College Station, TX, USA). We considered two-sided $p$-values below 0.05 as statistically significant.

\section{Results}

Table 1 summarizes selected baseline characteristics of the study participants by quintiles of $\sum$ dAGEs. Participants in the highest quintile consumed more processed meats, cakes, biscuits, cereals and cereal products, and legumes and less fruit. They also tended to consume less sugar, confectionery, and alcohol.

In minimally adjusted models (Model 1 ), CML (HR comparing highest to lowest quintile, $\mathrm{HR}_{\mathrm{Q} 5 \mathrm{vs} \cdot \mathrm{Q} 1}=0.83,95 \% \mathrm{CI}=0.77-0.90, p$ for trend $\left.<0.001\right)$ and $\mathrm{MG}-\mathrm{H} 1\left(\mathrm{HR}_{\mathrm{Q} 5 \mathrm{vs} \cdot} \cdot \mathrm{Q} 1=0.84\right.$, $95 \% \mathrm{CI}=0.77-0.90, p$ for trend $<0.001$ ) showed inverse associations with $\mathrm{CRC}$, whereas no significant association was observed for CEL $\left(\mathrm{HR}_{\mathrm{Q} 5 \mathrm{vs}} \cdot \mathrm{Q} 1=0.92,95 \% \mathrm{CI}=0.85-1.00\right.$, $p$ for trend $=0.064)($ Table 2). The significance of the associations was attenuated after full adjustment (Model 3), with HR (95\% CI) of 0.97 (0.94-0.99) and 0.97 (0.95-1.00) for CML and MG-H1, respectively.

Analyses by tumor subsites showed no significant heterogeneity between colon and rectal cancers, although the association with rectal cancer was statistically significant for CML (HR per $\ln (\mathrm{SD})$ increment: rectal cancer $\mathrm{HR}_{\ln (\mathrm{SD})}=0.93,95 \% \mathrm{CI}=0.88-0.97$ ) and MG-H1 (rectal cancer $\mathrm{HR}_{\ln (\mathrm{SD})}=0.94,95 \% \mathrm{CI}=0.90-0.99$ ) (Table 3). No significant difference in the association between the individual dAGEs and CRC risk was observed by sex (Supplementary Table S1). In stratified analyses, dAGEs-CRC risk did not differ by country, and tended to be restricted to participants with BMI $<30 \mathrm{~kg} / \mathrm{m}^{2}$ (Supplementary Table S2). When the follow-up time of cases was considered, dAGEs-CRC showed a gradient in the association between higher CRC risk observed and lower follow-up. Excluding participants with follow-up less than 2 years did not materially change the results (not shown). 
Table 1. Selected characteristics of the study participants (by quintiles of dietary AGEs), EPIC cohort study, $1992-2014$.

\begin{tabular}{|c|c|c|c|c|c|}
\hline & \multicolumn{5}{|c|}{ Quintiles of $\sum$ AGEs Intake } \\
\hline & Quintile 1 & Quintile 2 & Quintile 3 & Quintile 4 & Quintile 5 \\
\hline \multicolumn{6}{|l|}{ Recruitment and follow-up } \\
\hline Age at recruitment, years & $50.9 \pm 9.9$ & $50.5 \pm 9.7$ & $50.2 \pm 9.7$ & $50.1 \pm 9.7$ & $51.5 \pm 9.7$ \\
\hline Follow-up, years & $14.1 \pm 4.2$ & $14.1 \pm 4$ & $14.1 \pm 3.9$ & $14.1 \pm 3.8$ & $14.2 \pm 4.1$ \\
\hline \multicolumn{6}{|l|}{ Anthropometry } \\
\hline $\mathrm{BMI}, \mathrm{kg} / \mathrm{m}^{2}$ & $25.2 \pm 4.2$ & $25.3 \pm 4.2$ & $25.3 \pm 4.2$ & $25.3 \pm 4.2$ & $25.1 \pm 4.2$ \\
\hline \multicolumn{6}{|l|}{ Socio-demographic and lifestyle * } \\
\hline \multicolumn{6}{|l|}{ Education status, $\%$} \\
\hline None & 3.3 & 3.5 & 3.5 & 3.7 & 3.6 \\
\hline Primary school & 25.5 & 24.9 & 24.9 & 24.8 & 25.0 \\
\hline Technical or professional & 23.0 & 24.0 & 23.9 & 23.3 & 22.7 \\
\hline Secondary school & 20.6 & 20.6 & 21.3 & 22.3 & 21.0 \\
\hline Higher education & 24.7 & 24.4 & 24.3 & 24.2 & 25.1 \\
\hline \multicolumn{6}{|l|}{ Smoking status, \% } \\
\hline Never & 38.2 & 41.4 & 43.0 & 44.1 & 46.0 \\
\hline Current, $1-<16$ cigarettes/day & 12.8 & 12.3 & 11.9 & 11.5 & 9.58 \\
\hline Current, $16-<=20$ cigarettes / day & 8.1 & 6.75 & 6.1 & 5.5 & 4.19 \\
\hline Current, $>20$ cigarettes/day & 2.4 & 1.57 & 1.3 & 1.2 & 0.88 \\
\hline Former, quit $<=10$ years & 9.7 & 9.89 & 9.7 & 9.7 & 9.15 \\
\hline Former, quit $11-<20$ years & 8.1 & 8.42 & 8.4 & 8.4 & 8.56 \\
\hline Former, quit >20 years & 7.8 & 7.83 & 8.1 & 8.0 & 9.22 \\
\hline Current, pipe-cigar-occasional & 9.5 & 8.46 & 8.5 & 8.6 & 9.31 \\
\hline \multicolumn{6}{|l|}{ Physical activity status, $\%$} \\
\hline Inactive & 21.2 & 19.8 & 19.2 & 18.6 & 19.0 \\
\hline Moderately inactive & 33.3 & 33.7 & 33.3 & 32.9 & 33.4 \\
\hline Moderately active & 25.2 & 26.7 & 27.0 & 27.5 & 27.0 \\
\hline Active & 18.5 & 18.0 & 18.4 & 18.5 & 19.0 \\
\hline \multicolumn{6}{|l|}{ Daily dietary intake } \\
\hline Energy intake, kcal & $2052 \pm 775$ & $2084 \pm 639$ & $2091 \pm 585$ & $2092 \pm 548$ & $2063 \pm 512$ \\
\hline Red meat, $g$ & $44.4 \pm 40.3$ & $44.6 \pm 37$ & $43.3 \pm 35.2$ & $42.0 \pm 34.1$ & $38.9 \pm 33.1$ \\
\hline Processed meat, $g$ & $30.6 \pm 30.7$ & $33.1 \pm 30$ & $34.1 \pm 30.2$ & $34.1 \pm 30.1$ & $34.7 \pm 32.6$ \\
\hline Fibre, $\mathrm{g}$ & $20.1 \pm 8.1$ & $22.0 \pm 7.4$ & $23.1 \pm 7.2$ & $23.9 \pm 7.3$ & $25.1 \pm 7.9$ \\
\hline Dairy products, $\mathrm{g}$ & $345 \pm 271$ & $332 \pm 237$ & $326 \pm 229$ & $324 \pm 224$ & $341 \pm 226$ \\
\hline Fish and shellfish, $\mathrm{g}$ & $36.7 \pm 37.2$ & $38.6 \pm 37.1$ & $38.4 \pm 36.7$ & $37.6 \pm 35.9$ & $38.2 \pm 34.7$ \\
\hline Cakes and biscuits, $\mathrm{g}$ & $29.0 \pm 34.9$ & $39.3 \pm 40.5$ & $45.0 \pm 43.4$ & $49.2 \pm 45.8$ & $47.9 \pm 46.5$ \\
\hline Cereal and cereal products, $g$ & $171 \pm 101$ & $207 \pm 105$ & $224 \pm 106$ & $237 \pm 108$ & $260 \pm 121$ \\
\hline Fruits, nuts, and seeds, g & $242 \pm 220$ & $235 \pm 183$ & $231 \pm 169$ & $228 \pm 160$ & $225 \pm 156$ \\
\hline Vegetables, $\mathrm{g}$ & $205 \pm 146$ & $198 \pm 127$ & $193 \pm 122$ & $190 \pm 121$ & $200 \pm 128$ \\
\hline Legumes, g & $10.2 \pm 18.7$ & $13.2 \pm 21.7$ & $14.8 \pm 23.7$ & $16.2 \pm 25.7$ & $16.8 \pm 27.2$ \\
\hline Potatoes and other tubers, $g$ & $100 \pm 86.3$ & $96 \pm 75.1$ & $92.0 \pm 69.8$ & $89 \pm 68.3$ & $93 \pm 69.2$ \\
\hline Egg and egg products, $g$ & $17.6 \pm 18.7$ & $18.2 \pm 17$ & $18.2 \pm 16.5$ & $18.1 \pm 16.6$ & $17.8 \pm 17.1$ \\
\hline Fat, $g$ & $79.9 \pm 35.5$ & $81.3 \pm 29.8$ & $81.1 \pm 27.6$ & $80.6 \pm 26.5$ & $78.4 \pm 25.9$ \\
\hline Sugar and confectionery, $g$ & $50.3 \pm 76.3$ & $44.3 \pm 45.4$ & $41.8 \pm 38.7$ & $39.8 \pm 36.2$ & $37.6 \pm 33.4$ \\
\hline Alcohol, g & $18.9 \pm 25$ & $13.1 \pm 16.5$ & $10.9 \pm 14.1$ & $9.3 \pm 12.5$ & $8.3 \pm 11.3$ \\
\hline \multicolumn{6}{|l|}{ Mediterranean diet score, $\%$} \\
\hline Low & 32.8 & 26.2 & 23.9 & 22.8 & 21.2 \\
\hline Medium & 44.6 & 46.5 & 47.1 & 47.9 & 49.3 \\
\hline High & 22.6 & 27.3 & 28.9 & 29.3 & 29.6 \\
\hline
\end{tabular}

Abbreviation: AGE, advanced glycation end-products; EPIC, European Prospective Investigation into Cancer and Nutrition; mean \pm standard deviation is presented, unless otherwise stated; quintiles were calculated using total energy, center-standardized residuals, and log-transformed $\sum$ AGEs values. ${ }^{*}$ Percentages do not add up to $100 \%$ because of missing values. 
Table 2. Hazard ratios and 95\% confidence intervals (CI) for colorectal cancer risk associated with individual dietary AGEs (quintiles and continuous), EPIC cohort study, 1992-2014.

\begin{tabular}{|c|c|c|c|c|c|}
\hline Dietary AGE & $N$ Cases & Median Intake & Model 1 & Model 2 & Model 3 \\
\hline \multicolumn{6}{|l|}{$\mathrm{CML}, \mathrm{mg} /$ day } \\
\hline Quintile 1 & 1391 & 1.90 & 1.00 (Ref.) & 1.00 (Ref.) & 1.00 (Ref.) \\
\hline Quintile 2 & 1259 & 2.41 & $0.93(0.86-1.00)$ & $0.94(0.87-1.02)$ & $0.98(0.90-1.06)$ \\
\hline Quintile 3 & 1210 & 2.75 & $0.91(0.85-0.99)$ & $0.94(0.87-1.01)$ & $0.98(0.91-1.07)$ \\
\hline Quintile 4 & 1120 & 3.16 & $0.85(0.79-0.92)$ & $0.88(0.81-0.95)$ & $0.93(0.86-1.01)$ \\
\hline Quintile 5 & 1182 & 4.02 & $0.83(0.77-0.90)$ & $0.87(0.80-0.94)$ & $0.92(0.85-1.00)$ \\
\hline$p$ for trend & & & $<0.001$ & $<0.001$ & 0.023 \\
\hline per $\ln (\mathrm{SD})$ increase & & & $0.94(0.91-0.96)$ & $0.95(0.92-0.97)$ & $0.97(0.94-0.99)$ \\
\hline \multicolumn{6}{|l|}{ CEL, mg/day } \\
\hline Quintile 1 & 1214 & 1.37 & 1.00 (Ref.) & 1.00 (Ref.) & 1.00 (Ref.) \\
\hline Quintile 2 & 1271 & 1.71 & $1.00(0.92-1.08)$ & $1.00(0.92-1.08)$ & $1.02(0.95-1.11)$ \\
\hline Quintile 3 & 1219 & 1.93 & $0.95(0.88-1.03)$ & $0.95(0.88-1.03)$ & $0.99(0.91-1.07)$ \\
\hline Quintile 4 & 1268 & 2.21 & $0.99(0.91-1.07)$ & $1.00(0.92-1.08)$ & $1.04(0.96-1.13)$ \\
\hline Quintile 5 & 1190 & 2.85 & $0.92(0.85-1.00)$ & $0.92(0.85-1.00)$ & $0.97(0.89-1.05)$ \\
\hline$p$ for trend & & & 0.064 & 0.072 & 0.630 \\
\hline per $\ln (\mathrm{SD})$ increase & & & $0.97(0.95-1.00)$ & $0.97(0.95-1.00)$ & $0.99(0.96-1.01)$ \\
\hline \multicolumn{6}{|l|}{ MG-H1, mg/day } \\
\hline Quintile 1 & 1388 & 13.0 & 1.00 (Ref.) & 1.00 (Ref.) & 1.00 (Ref.) \\
\hline Quintile 2 & 1250 & 16.7 & $0.94(0.87-1.01)$ & $0.94(0.87-1.02)$ & $0.98(0.90-1.06)$ \\
\hline Quintile 3 & 1183 & 19.3 & $0.91(0.84-0.99)$ & $0.93(0.86-1.00)$ & $0.97(0.90-1.06)$ \\
\hline Quintile 4 & 1120 & 22.5 & $0.88(0.81-0.95)$ & $0.89(0.82-0.97)$ & $0.94(0.87-1.03)$ \\
\hline Quintile 5 & 1221 & 29.9 & $0.84(0.77-0.90)$ & $0.86(0.80-0.94)$ & $0.92(0.85-1.00)$ \\
\hline$p$ for trend & & & $<0.001$ & $<0.001$ & 0.033 \\
\hline per $\ln (\mathrm{SD})$ increase & & & $0.94(0.92-0.97)$ & $0.95(0.93-0.98)$ & $0.97(0.95-1.00)$ \\
\hline
\end{tabular}

Abbreviations: AGE, advanced glycation end-product; CML, Ne-carboxy-methyllysine; CEL, Ne-carboxy-ethyllysine; EPIC, European Prospective Investigation into Cancer and Nutrition; MG-H1, N8-(5-hydro-5-methyl-4-imidazolon-2-yl)-ornithine; Model 1 is stratified by age (1-year categories), sex, and center; Model 2 is additionally adjusted for BMI, height, education, physical activity, smoking, and energy intake; Model 3 is Model 2 additionally adjusted for the Mediterranean diet score.

Table 3. Hazard ratios and 95\% confidence intervals (CI) for the risk of colorectal cancer in anatomical subsites associated with dietary AGEs (quintiles and continuous), EPIC cohort study, 1992-2014.

\begin{tabular}{|c|c|c|c|c|c|c|c|c|c|}
\hline & \multirow{3}{*}{$\begin{array}{l}\text { Median } \\
\text { Intake }\end{array}$} & \multicolumn{6}{|c|}{ Colon Cancer } & \multicolumn{2}{|c|}{ Rectal Cancer } \\
\hline & & \multicolumn{2}{|r|}{ All } & \multicolumn{2}{|c|}{ Proximal Colon } & \multicolumn{2}{|c|}{ Distal Colon } & \multirow[b]{2}{*}{$\begin{array}{c}N \\
\text { Cases }\end{array}$} & \multirow[b]{2}{*}{ HR (95\% CI ) } \\
\hline & & $\begin{array}{c}N \\
\text { Cases }\end{array}$ & HR (95\% CI ) & $\begin{array}{c}N \\
\text { Cases }\end{array}$ & HR (95\% CI ) & $\begin{array}{c}N \\
\text { Cases }\end{array}$ & HR (95\% CI ) & & \\
\hline \multicolumn{10}{|l|}{ CML, mg/day } \\
\hline Quintile 1 & 1.90 & 873 & 1.00 (Ref.) & 399 & 1.00 (Ref.) & 397 & 1.00 (Ref.) & 518 & 1.00 (Ref.) \\
\hline Quintile 2 & 2.41 & 774 & $\begin{array}{c}0.95 \\
(0.86-1.05)\end{array}$ & 357 & $\begin{array}{c}0.95 \\
(0.82-1.10)\end{array}$ & 317 & $\begin{array}{c}0.87 \\
(0.75-1.02)\end{array}$ & 485 & $\begin{array}{c}1.02 \\
(0.90-1.17)\end{array}$ \\
\hline Quintile 3 & 2.75 & 786 & $\begin{array}{c}1.01 \\
(0.91-1.11)\end{array}$ & 366 & $\begin{array}{c}1.01 \\
(0.87-1.17)\end{array}$ & 319 & $\begin{array}{c}0.92 \\
(0.79-1.08)\end{array}$ & 424 & $\begin{array}{c}0.95 \\
(0.83-1.09)\end{array}$ \\
\hline Quintile 4 & 3.16 & 759 & $\begin{array}{c}0.99 \\
(0.89-1.09)\end{array}$ & 345 & $\begin{array}{c}0.95 \\
(0.82-1.11)\end{array}$ & 351 & $\begin{array}{c}1.04 \\
(0.89-1.21)\end{array}$ & 361 & $\begin{array}{c}0.81 \\
(0.70-0.94)\end{array}$ \\
\hline Quintile 5 & 4.02 & 805 & $\begin{array}{c}0.98 \\
(0.89-1.09)\end{array}$ & 389 & $\begin{array}{c}0.99 \\
(0.85-1.15)\end{array}$ & 342 & $\begin{array}{c}0.97 \\
(0.83-1.13)\end{array}$ & 377 & $\begin{array}{c}0.81 \\
(0.70-0.93)\end{array}$ \\
\hline$p$ for trend & & & 0.989 & & 0.944 & & 0.564 & & $<0.001$ \\
\hline & & & 0.99 & & 1.00 & & 0.99 & & 0.93 \\
\hline per In(SD) increase & & & $(0.96-1.02)$ & & $(0.95-1.05)$ & & $(0.94-1.04)$ & & $(0.88-0.97)$ \\
\hline \multicolumn{10}{|l|}{ CEL, mg/day } \\
\hline Quintile 1 & 1.37 & 763 & 1.00 (Ref.) & 336 & 1.00 (Ref.) & 357 & 1.00 (Ref.) & 451 & 1.00 (Ref.) \\
\hline Quintile 2 & 1.71 & 830 & $\begin{array}{c}1.06 \\
(0.95-1.17)\end{array}$ & 380 & $\begin{array}{c}1.10 \\
(0.94-1.27)\end{array}$ & 365 & $\begin{array}{c}1.00 \\
(0.86-1.16)\end{array}$ & 441 & $\begin{array}{c}0.99 \\
(0.86-1.13)\end{array}$ \\
\hline Quintile 3 & 1.93 & 789 & $\begin{array}{c}1.01 \\
(0.91-1.12)\end{array}$ & 348 & $\begin{array}{c}1.02 \\
(0.87-1.19)\end{array}$ & 353 & $\begin{array}{c}0.98 \\
(0.84-1.14)\end{array}$ & 430 & $\begin{array}{c}0.94 \\
(0.82-1.08)\end{array}$ \\
\hline
\end{tabular}


Table 3. Cont.

\begin{tabular}{|c|c|c|c|c|c|c|c|c|c|}
\hline & \multirow{3}{*}{$\begin{array}{l}\text { Median } \\
\text { Intake }\end{array}$} & \multicolumn{6}{|c|}{ Colon Cancer } & \multicolumn{2}{|c|}{ Rectal Cancer } \\
\hline & & \multirow[b]{2}{*}{$\begin{array}{c}N \\
\text { Cases }\end{array}$} & All & \multicolumn{2}{|c|}{ Proximal Colon } & \multicolumn{2}{|c|}{ Distal Colon } & \multirow[b]{2}{*}{$\begin{array}{c}N \\
\text { Cases }\end{array}$} & \multirow[b]{2}{*}{ HR (95\% CI) } \\
\hline & & & HR (95\% CI ) & $\begin{array}{c}N \\
\text { Cases }\end{array}$ & HR $(95 \%$ CI ) & $\begin{array}{c}N \\
\text { Cases }\end{array}$ & HR $(95 \%$ CI ) & & \\
\hline Quintile 4 & 2.21 & 828 & $\begin{array}{c}1.08 \\
(0.97-1.19)\end{array}$ & 402 & $\begin{array}{c}1.18 \\
(1.01-1.37)\end{array}$ & 323 & $\begin{array}{c}0.92 \\
(0.79-1.07)\end{array}$ & 440 & $\begin{array}{c}0.99 \\
(0.86-1.14)\end{array}$ \\
\hline Quintile 5 & 2.85 & 787 & $\begin{array}{c}1.01 \\
(0.91-1.12)\end{array}$ & 390 & $\begin{array}{c}1.12 \\
(0.96-1.31)\end{array}$ & 328 & $\begin{array}{c}0.93 \\
(0.79-1.08)\end{array}$ & 403 & $\begin{array}{c}0.89 \\
(0.77-1.03)\end{array}$ \\
\hline$p$ for trend & & & 0.697 & & 0.083 & & 0.194 & & 0.166 \\
\hline per $\ln (\mathrm{SD})$ increase & & & $\begin{array}{c}1.00 \\
(0.97-1.04)\end{array}$ & & $\begin{array}{c}1.03 \\
(0.99-1.09)\end{array}$ & & $\begin{array}{c}0.97 \\
(0.92-1.02)\end{array}$ & & $\begin{array}{c}0.96 \\
(0.92-1.00)\end{array}$ \\
\hline MG-H1, mg/day & & & & & & & & & \\
\hline Quintile 1 & 13.0 & 861 & 1.00 (Ref.) & 384 & 1.00 (Ref.) & 386 & 1.00 (Ref.) & 527 & 1.00 (Ref.) \\
\hline Quintile 2 & 16.7 & 806 & $\begin{array}{c}1.00 \\
(0.91-1.10)\end{array}$ & 367 & $\begin{array}{c}1.02 \\
(0.88-1.19)\end{array}$ & 352 & $\begin{array}{c}0.98 \\
(0.84-1.13)\end{array}$ & 444 & $\begin{array}{c}0.95 \\
(0.83-1.09)\end{array}$ \\
\hline Quintile 3 & 19.3 & 770 & $\begin{array}{c}1.01 \\
(0.91-1.11)\end{array}$ & 351 & $\begin{array}{c}1.02 \\
(0.87-1.18)\end{array}$ & 342 & $\begin{array}{c}1.01 \\
(0.86-1.17)\end{array}$ & 413 & $\begin{array}{c}0.93 \\
(0.81-1.07)\end{array}$ \\
\hline Quintile 4 & 22.5 & 735 & $\begin{array}{c}0.98 \\
(0.89-1.09)\end{array}$ & 348 & $\begin{array}{c}1.03 \\
(0.89-1.20)\end{array}$ & 306 & $\begin{array}{c}0.92 \\
(0.78-1.08)\end{array}$ & 385 & $\begin{array}{c}0.89 \\
(0.77-1.02)\end{array}$ \\
\hline Quintile 5 & 29.9 & 825 & $\begin{array}{c}0.99 \\
(0.90-1.10)\end{array}$ & 406 & $\begin{array}{c}1.06 \\
(0.91-1.23)\end{array}$ & 340 & $\begin{array}{c}0.94 \\
(0.81-1.10)\end{array}$ & 396 & $\begin{array}{c}0.81 \\
(0.71-0.94)\end{array}$ \\
\hline$p$ for trend & & & 0.793 & & 0.457 & & 0.326 & & 0.003 \\
\hline per $\ln (S D)$ increase & & & $\begin{array}{c}0.99 \\
(0.96-1.02)\end{array}$ & & $\begin{array}{c}1.03 \\
(0.98-1.07)\end{array}$ & & $\begin{array}{c}0.96 \\
(0.92-1.01)\end{array}$ & & $\begin{array}{c}0.94 \\
(0.90-0.99)\end{array}$ \\
\hline
\end{tabular}

Abbreviations: AGE, advanced glycation end-product; CI, confidence interval; CML, Ne-carboxy-methyllysine; CEL, Ne-carboxyethyllysine; EPIC, European Prospective Investigation into Cancer and Nutrition; HR, hazard ratio; MG-H1, N $\delta$-(5-hydro-5-methyl4-imidazolon-2-yl)-ornithine; models were adjusted for body mass index, height, education, physical activity, smoking, energy intake and Mediterranean diet score and stratified by age (1-year categories), sex, and center; $p$ for heterogeneity between colon and rectal cancer was $0.391,0.849$, and 0.825 for CML, CEL, and MG-H1, respectively; $p$ for heterogeneity between proximal and distal colon cancer was 0.878 , 0.793 , and 0.804 for CML, CEL, and MG-H1, respectively.

\section{Discussion}

In this large prospective study, we found that dietary intakes of CML and MG-H1, but not CEL, were inversely associated with the risk of CRC. Our analyses did not identify any heterogeneity in these findings by anatomical subsite of the tumor within the colorectum, by sex, or by follow-up time.

Our findings were contrary to our initial hypothesis that dAGE exposure could promote CRC development. This hypothesis was based on considerable experimental evidence suggesting cancer-promoting characteristics for these compounds. Three main mechanisms have been postulated: first, AGEs may bind to the RAGE receptor in colonocytes and, subsequently, promote and sustain inflammation and oxidative stress [29,30]; second, they may modify the composition of the microbiome towards microbial genera that are deleterious to gut health [31]; and finally, they may increase gut permeability, thereby allowing bacterial translocation and increased exposure of colonocytes to toxic bacterial compounds [32]. In in vitro enterocyte models, cells treated with AGEs have shown higher expression of RAGE and an increase in inflammatory factors, such as IL-8, IL-1 $\beta$, and nuclear factor-kappa B (NF-kB) [13,33], suggesting that dAGEs may produce similar effects in the gut. Nevertheless, a main condition for this to occur in vivo is that CML, CEL, and MG-H1 need to reach the colon untransformed, in a protein-bound form which could be recognized by RAGE and be able to interact with the cell surface of colonocytes. This is because several studies have reported that free AGEs or those attached to single amino acids are not as recognizable by RAGE as protein-bound AGEs [34,35]. A recent study using a dynamic in vitro model showed that protein-bound dAGEs can survive intestinal digestion and remain in the gastrointestinal tract [36]. Zenker et al. [37], using a model with casein, have shown in a recent study that unglycated proteins could also interact with RAGE. This provides additional evidence supporting the complexity of the AGEs, particularly their interactions in the gastrointestinal milieu. It is evident that more knowledge is needed on 
the role of the microbiome and intestinal conditions in the conservation or degradation of protein-bound dAGEs, and how this may affect RAGE-specific inflammation.

Recent growing evidence suggested that the human gut microbiome can metabolize dAGEs, possibly as much as $40 \%$ for ingested CML [38]. CML has been shown to be metabolized by the microbiome into several sub-products, including biogenic amines and fatty acids, notably $\mathrm{N}$-carboxymethylcadaverine, $\mathrm{N}$-carboxymethylaminopentanoic acid, $\mathrm{N}$-carboxymethyl- $\Delta$ 1-piperideinium ion, and 2-amino-6-(formylmethylamino)hexanoic acid $[39,40]$. Less is known about the specific actions of these catabolic products within the colorectum, or the possible downstream molecules that can be produced from them. It is possible that these compounds may not be recognized by RAGE, and, hence, not induce an inflammatory response within the gut. Nevertheless, dAGEs have been associated with reduced diversity and richness of the gut microbiome, which is thought to be conducive to a CRC-promoting environment [15]. Thus, the possible microbial metabolism of dAGEs may not entirely negate their deleterious properties. It is also noteworthy that the three dAGEs that we assessed are thought to bind to a single domain of RAGE (V domain), whereas other AGE compounds, such as pentosidine, which are much less abundant in the diet, could bind to additional domains ( $\mathrm{V}$ and $\mathrm{C} 1$ domains) [41], potentially triggering a stronger inflammatory response. This suggests that future studies should also consider studying the potential deleterious properties of other less abundant dAGEs and their possible CRC risk associations.

We are unsure why we observed inverse CRC risk associations with CML and MGH1, whereas CEL demonstrated no association. One possible explanation may relate to the different dietary sources for these compounds. In EPIC, CML and MG-H1 share very similar food sources (e.g., mostly cereals and cereal products), while CEL is derived to a greater extent from meats [23,42]. Cereals and cereal products are major sources of dietary fiber, which has been previously associated with lower CRC risk and could partially explain the inverse association observed with CML and MG-H1 [43]. Another potential explanation for the differential CRC risk associations for these compounds may be the chemical pathways through which they are produced. Although the three AGEs are all likely derived from reducing sugars, their main precursors are reactive dicarbonyl compounds, particularly glyoxal (GO) and methylglyoxal (MGO). CML originates from GO, whereas CEL and MG-H1 are mainly produced from MGO [44]. In addition, CEL and MG-H1 (the two MGO-derived AGEs) differ in their amino acid content, as CEL is produced from lysine, while MG-H1 derives from arginine [45]. Interestingly, reactive dicarbonyl compounds are thought to possess a glycating potential that may be thousands of times higher compared to that of sugars such as glucose or fructose [46] and, hence, the CRC risk association of these dietary compounds and their precursors may also warrant further study.

The importance of studying dAGEs in CRC development lies in several potential public health measures that may be taken to control their exposures at wider population levels. For example, dAGEs may be directly targeted through adoption of specific cooking methods (e.g., steaming) to reduce the dAGE content of specific processed foods, or possibly with anti-glycation dietary compounds (e.g., polyphenols) [47] to counter any possible adverse effects [48,49].

Although, to date, no large prospective studies have explored the association between dAGEs and CRC development, the role of circulating AGEs in CRC development has been explored in three separate case-control studies nested within prospective cohorts, with discordant findings. Two of the three studies reported inverse associations with CRC [50,51], whereas one reported a positive association in male smokers [52]. It is noteworthy that, among these previous studies, two [50,52] estimated AGEs using ELISA, which is now recognized as providing biased AGE estimation [53]. Nevertheless, taken together with our study, these observations suggest that the role of AGEs in CRC development is likely to be quite complex, and further studies of other AGE compounds beyond the three studied here are also warranted. 
The strengths of our study include its large study population, its prospective design, and the large number of CRC cases, which allowed us to run extensive analysis and control for a comprehensive number of confounders. In addition, our study followed the recent "quality control" recommendations for studies on AGEs, i.e., the study of several specific AGEs and the use of a validated food composition database to estimate individual dAGE exposures [54]. However, our study was limited by the single collection of dAGEs and other covariates at baseline; thus, potential changes in diet or covariates during follow-up could not be accounted for. AGE levels in the foods are influenced by cooking methods, i.e., frying, baking, or broiling, and conditions, such as cooking temperature, humidity, and $\mathrm{pH}$ [55]. Hence, it is possible that country-specific differences in cooking conditions and varying geographical and/or individual preferences for doneness of similar foods items could have impacted our dietary AGE estimation. Additionally, it is possible that residual confounding cannot be completely ruled out.

In conclusion, we found inverse associations between the intake of CML and of MG-H1, but not of CEL, and the risk of CRC in the large EPIC prospective cohort. Our findings corroborate some previous findings from circulating AGEs, suggesting that the three AGEs included in our study may not be CRC-promotive, as has previously been suspected. Our study provides additional evidence of the complexity of AGEs and their interaction in CRC and calls for additional studies to confirm our findings and to explore the link between CRC and other dAGEs not studied herein.

Supplementary Materials: The following are available online at https: / www.mdpi.com/article / 10.3390/nu13093132/s1, Supplementary Table S1: Hazard ratios and 95\% confidence intervals (CI) for colorectal cancer risk associated with AGEs intake, by sex, EPIC cohort study, 1992-2014; Supplementary Table S2: Stratified analysis for colorectal cancer risk associated with AGEs intake by BMI, years of follow-up and by EPIC country; Supplementary Figure S1: Dietary contribution of individual advanced glycation end-products in the European Prospective Investigation into Cancer and Nutrition (EPIC).

Author Contributions: Conceptualization, M.J., H.F., C.G.S., and V.F.; methodology, M.J., V.K. (Viktoria Knaze), H.F.; software, E.K.A.; validation, V.K. (Verena Katzke), V.F., A.-L.M., formal analysis, E.K.A.; investigation, E.K.A., A.-L.M., V.K. (Viktoria Knaze), H.F., V.F., D.J.H., L.J., A.K.E., A.T., M.-C.B.-R., J.A.R., G.S. (Gianluca Sever), R.K., V.K. (Verena Katzke), M.B.S., A.B., D.P., S.S., M.S.d.M., R.T., F.R., B.B.-d.-M., J.W.G.D., G.S. (Guri Skeie), I.T.G., T.S., J.R.Q., L.L.-B., M.-J.S., P.A., M.-D.C., A.B.G., I.J., J.M., A.P.-C., E.W., M.J.G., A.K.H., C.G.S., M.J.; resources, E.K.A., A.-L.M., V.K. (Viktoria Knaze), H.F., V.F., D.J.H., L.J., A.K.E., A.T., M.-C.B.-R., J.A.R., G.S. (Gianluca Sever), R.K., V.K. (Verena Katzke), M.B.S., A.B., D.P., S.S., M.S.d.M., R.T., F.R., B.B.-d.-M., J.W.G.D., G.S. (Guri Skeie), I.T.G., T.S., J.R.Q., L.L.-B., M.-J.S., P.A., M.-D.C., A.B.G., I.J., J.M., A.P.-C., E.W., M.J.G., A.K.H., C.G.S., M.J.; data curation, E.K.A.; writing - original draft preparation, E.K.A.; writing-review and editing, E.K.A., A.-L.M., V.K. (Viktoria Knaze), H.F., V.F., D.J.H., L.J., A.K.E., A.T., M.-C.B.-R., J.A.R., G.S. (Gianluca Sever), R.K., V.K. (Verena Katzke), M.B.S., A.B., D.P., S.S., M.S.d.M., R.T., F.R., B.B.-d.-M., J.W.G.D., G.S. (Guri Skeie), I.T.G., T.S., J.R.Q., L.L.-B., M.-J.S., P.A., M.-D.C., A.B.G., I.J., J.M., A.P.-C., E.W., M.J.G., A.K.H., C.G.S., M.J.; supervision, M.J.; project administration, M.J.; funding, M.J. All authors have read and agreed to the published version of the manuscript.

Funding: This work was funded by the Wereld Kanker Onderzoek Fonds (WKOF), as part of the World Cancer Research Fund (WCRF) International grant program (WCRF 2015-1391, P.I. Mazda Jenab, International Agency for Research on Cancer). This work was partially financially supported by the Fondation de France (FDF grant no. 00081166 to H. Freisling and FDF grant no. 00089811 to A.-L. Mayén). The funders had no role in study design, data collection and analysis, decision to publish, or preparation of the manuscript.

Institutional Review Board Statement: The study was conducted according to the guidelines of the Declaration of Helsinki, and approved by the Ethical Committee of the International Agency for Research on Cancer (IARC) and local ethical committees pertaining to EPIC Centers. The EPIC cohort was successfully reevaluated by the International Agency for Research on Cancer Ethics Committee in 2017. The current study received further approval by the IARC Ethics Committee (IEC Project No. 18-10). 
Informed Consent Statement: Informed consent was obtained from all subjects involved in the study.

Data Availability Statement: Data described in the study, code book and analytic code will be made available upon request. For information on how to submit an application for gaining access to EPIC data and/or biospecimens, please follow the instructions at http:/ / epic.iarc.fr/access/index.php.

Acknowledgments: The authors would like to thank the EPIC study participants and staff for their valuable contribution to this research. The authors would also like to thank Bertrand Hemon for the preparation of the databases. The coordination of EPIC is financially supported by the International Agency for Research on Cancer (IARC) and also by the Department of Epidemiology and Biostatistics, School of Public Health, Imperial College London, which has additional infrastructure support provided by the NIHR Imperial Biomedical Research Centre (BRC). The national cohorts are supported by: Danish Cancer Society (Denmark); Ligue Contre le Cancer, Institut Gustave Roussy, Mutuelle Générale de l'Education Nationale, Institut National de la Santé et de la Recherche Médicale (INSERM) (France); German Cancer Aid, German Cancer Research Center (DKFZ), German Institute of Human Nutrition Potsdam-Rehbruecke (DIfE), Federal Ministry of Education and Research (BMBF) (Germany); Associazione Italiana per la Ricerca sul Cancro (AIRC), Compagnia di San Paolo, and National Research Council (Italy); Dutch Ministry of Public Health, Welfare and Sports (VWS), Netherlands Cancer Registry (NKR), LK Research Funds, Dutch Prevention Funds, Dutch ZON (Zorg Onderzoek Nederland), World Cancer Research Fund (WCRF), Statistics Netherlands (The Netherlands); Health Research Fund (FIS), Instituto de Salud Carlos III (ISCIII), Regional Governments of Andalucía, Asturias, the Basque Country, Murcia, and Navarra, and the Catalan Institute of Oncology (ICO) (Spain); Swedish Cancer Society, Swedish Research Council, and County Councils of Skåne and Västerbotten (Sweden); Cancer Research UK (14136 to EPIC-Norfolk; C8221/A29017 to EPIC-Oxford), Medical Research Council (1000143 to EPIC-Norfolk; MR/M012190/1 to EPIC-Oxford) (United Kingdom). We are grateful to all of the participants who have been part of the project and to the many members of the study teams at the University of Cambridge who have enabled this research. We acknowledge the use of data from the EPIC-Aarhus cohort, PI Kim Overvad. We thank the CERCA Programme/Generalitat de Catalunya for institutional support.

Conflicts of Interest: The authors declare no conflict of interest.

Disclaimer: Where authors are identified as personnel of the International Agency for Research on Cancer/World Health Organization, the authors alone are responsible for the views expressed in this article and they do not necessarily represent the decisions, policy, or views of the International Agency for Research on Cancer/World Health Organization.

\footnotetext{
Abbreviations

AGE: advanced glycation end-product; BMI, body mass index; CEL, $\mathrm{N}^{\varepsilon}$-(carboxyethyl)lysine; $\mathrm{CI}$, confidence interval; CML, $\mathrm{N}^{\varepsilon}$-(carboxymethyl)lysine; CRC, colorectal cancer; EPIC, European Prospective Investigation into Cancer and Nutrition; IARC, International Agency for Research on Cancer; ICD, international classification of diseases; MG-H1, N8-(5-hydro-5-methyl-4-imidazolon-2yl)-ornithine; RAGE, receptor for AGE; SD, standard deviation.
}

\section{References}

1. IARC. Global Cancer Observatory-Cancer Today; IARC-WHO: Lyon, France, 2020.

2. Fidler, M.M.; Soerjomataram, I.; Bray, F. A global view on cancer incidence and national levels of the human development index. Int. J. Cancer 2016, 139, 2436-2446. [CrossRef] [PubMed]

3. Safiri, S.; Sepanlou, S.G.; Ikuta, K.S.; Bisignano, C.; Salimzadeh, H.; Delavari, A.; Ansari, R.; Roshandel, G.; Merat, S.; Fitzmaurice, C.; et al. The global, regional, and national burden of colorectal cancer and its attributable risk factors in 195 countries and territories, 1990-2017: A systematic analysis for the Global Burden of Disease Study 2017. Lancet Gastroenterol. Hepatol. 2019, 4, 913-933. [CrossRef]

4. Murphy, N.; Moreno, V.; Hughes, D.J.; Vodicka, L.; Vodicka, P.; Aglago, E.K.; Gunter, M.J.; Jenab, M. Lifestyle and dietary environmental factors in colorectal cancer susceptibility. Mol. Asp. Med. 2019, 69, 2-9. [CrossRef]

5. Mehta, R.S.; Song, M.; Nishihara, R.; Drew, D.A.; Wu, K.; Qian, Z.R.; Fung, T.T.; Hamada, T.; Masugi, Y.; da Silva, A.; et al. Dietary Patterns and Risk of Colorectal Cancer: Analysis by Tumor Location and Molecular Subtypes. Gastroenterology 2017, 152, 1944-1953.e1. [CrossRef]

6. Fournet, M.; Bonté, F.; Desmoulière, A. Glycation Damage: A Possible Hub for Major Pathophysiological Disorders and Aging. Aging Dis. 2018, 9, 880-900. [CrossRef] [PubMed] 
7. Aragno, M.; Mastrocola, R. Dietary Sugars and Endogenous Formation of Advanced Glycation Endproducts: Emerging Mechanisms of Disease. Nutrients 2017, 9, 385. [CrossRef] [PubMed]

8. Moura, F.A.; Goulart, M.O.F.; Campos, S.B.G.; da Paz Martins, A.S. The Close Interplay of Nitro-Oxidative Stress, Advanced Glycation end Products and Inflammation in Inflammatory Bowel Diseases. Curr. Med. Chem. 2020, 27, 2059-2076. [CrossRef] [PubMed]

9. Somoza, V.; Wenzel, E.; Weiss, C.; Clawin-Rädecker, I.; Grübel, N.; Erbersdobler, H.F. Dose-dependent utilisation of casein-linked lysinoalanine, N(epsilon)-fructoselysine and N(epsilon)-carboxymethyllysine in rats. Mol. Nutr. Food Res. 2006, 50, 833-841. [CrossRef]

10. Koschinsky, T.; He, C.J.; Mitsuhashi, T.; Bucala, R.; Liu, C.; Buenting, C.; Heitmann, K.; Vlassara, H. Orally absorbed reactive glycation products (glycotoxins): An environmental risk factor in diabetic nephropathy. Proc. Natl. Acad. Sci. USA 1997, 94, 6474-6479. [CrossRef]

11. Henle, T. AGEs in foods: Do they play a role in uremia? Kidney Int. 2003, 63, S145-S147. [CrossRef]

12. Uribarri, J.; Cai, W.; Sandu, O.; Peppa, M.; Goldberg, T.; Vlassara, H. Diet-derived advanced glycation end products are major contributors to the body's AGE pool and induce inflammation in healthy subjects. Ann. N. Y. Acad. Sci. 2005, 1043, 461-466. [CrossRef]

13. Geicu, O.I.; Stanca, L.; Voicu, S.N.; Dinischiotu, A.; Bilteanu, L.; Serban, A.I.; Calu, V. Dietary AGEs involvement in colonic inflammation and cancer: Insights from an in vitro enterocyte model. Sci. Rep. 2020, 10, 2754. [CrossRef]

14. Sakellariou, S.; Fragkou, P.; Levidou, G.; Gargalionis, A.N.; Piperi, C.; Dalagiorgou, G.; Adamopoulos, C.; Saetta, A.; Agrogiannis, G.; Theohari, I.; et al. Clinical significance of AGE-RAGE axis in colorectal cancer: Associations with glyoxalase-I, adiponectin receptor expression and prognosis. BMC Cancer 2016, 16, 174. [CrossRef]

15. Qu, W.; Yuan, X.; Zhao, J.; Zhang, Y.; Hu, J.; Wang, J.; Li, J. Dietary advanced glycation end products modify gut microbial composition and partially increase colon permeability in rats. Mol. Nutr. Food Res. 2017, 61, 1700118. [CrossRef] [PubMed]

16. Takeuchi, M.; Takino, J.-I.; Furuno, S.; Shirai, H.; Kawakami, M.; Muramatsu, M.; Kobayashi, Y.; Yamagishi, S.-I. Assessment of the Concentrations of Various Advanced Glycation End-Products in Beverages and Foods That Are Commonly Consumed in Japan. PLoS ONE 2015, 10, e0118652. [CrossRef] [PubMed]

17. Scheijen, J.; Clevers, E.; Engelen, L.; Dagnelie, P.C.; Brouns, F.; Stehouwer, C.D.A.; Schalkwijk, C.G. Analysis of advanced glycation endproducts in selected food items by ultra-performance liquid chromatography tandem mass spectrometry: Presentation of a dietary AGE database. Food Chem. 2016, 190, 1145-1150. [CrossRef] [PubMed]

18. Riboli, E.; Hunt, K.J.; Slimani, N.; Ferrari, P.; Norat, T.; Fahey, M.; Charrondiere, U.R.; Hemon, B.; Casagrande, C.; Vignat, J.; et al. European Prospective Investigation into Cancer and Nutrition (EPIC): Study populations and data collection. Public Health Nutr. 2002, 5, 1113-1124. [CrossRef]

19. Slimani, N.; Casagrande, C.; Nicolas, G.; Freisling, H.; Huybrechts, I.; Ocke, M.C. The standardized computerized 24-h dietary recall method EPIC-Soft adapted for pan-European dietary monitoring. Eur. J. Clin. Nutr. 2011, 65 (Suppl. S1), S5-S15. [CrossRef] [PubMed]

20. Slimani, N.; Ferrari, P.; Ocké, M.; Welch, A.; Boeing, H.; Liere, M.; Pala, V.; Amiano, P.; Lagiou, A.; Mattisson, I.; et al. Standardization of the 24-hour diet recall calibration method used in the european prospective investigation into cancer and nutrition (EPIC): General concepts and preliminary results. Eur. J. Clin. Nutr. 2000, 54, 900-917. [CrossRef]

21. Slimani, N.; Deharveng, G.; Unwin, I.; Southgate, D.A.; Vignat, J.; Skeie, G.; Salvini, S.; Parpinel, M.; Moller, A.; Ireland, J.; et al. The EPIC nutrient database project (ENDB): A first attempt to standardize nutrient databases across the 10 European countries participating in the EPIC study. Eur. J. Clin. Nutr. 2007, 61, 1037-1056. [CrossRef]

22. Nicolas, G.; Witthoft, C.M.; Vignat, J.; Knaze, V.; Huybrechts, I.; Roe, M.; Finglas, P.; Slimani, N. Compilation of a standardised international folate database for EPIC. Food Chem. 2016, 193, 134-140. [CrossRef] [PubMed]

23. Mayén, A.L.; Aglago, E.K.; Knaze, V.; Cordova, R.; Schalkwijk, C.G.; Wagner, K.H.; Aleksandrova, K.; Fedirko, V.; Keski-Rahkonen, P.; Leitzmann, M.F.; et al. Dietary intake of advanced glycation endproducts and risk of hepatobiliary cancers: A multinational cohort study. Int. J. Cancer 2021, 149, 854-864. [CrossRef]

24. Jones, P.; Cade, J.E.; Evans, C.E.L.; Hancock, N.; Greenwood, D.C. The Mediterranean diet and risk of colorectal cancer in the UK Women's Cohort Study. Int. J. Epidemiol. 2017, 46, 1786-1796. [CrossRef] [PubMed]

25. Harrell, F.E., Jr. Package 'rms'; The Comprehensive R Archive Network: Vienna, Austria, 2016.

26. Desquilbet, L.; Mariotti, F. Dose-response analyses using restricted cubic spline functions in public health research. Stat. Med. 2010, 29, 1037-1057. [CrossRef] [PubMed]

27. Lunn, M.; McNeil, D. Applying Cox regression to competing risks. Biometrics 1995, 51, 524-532. [CrossRef]

28. Scrucca, L.; Santucci, A.; Aversa, F. Competing risk analysis using R: An easy guide for clinicians. Bone Marrow Transpl. 2007, 40, 381-387. [CrossRef] [PubMed]

29. Ott, C.; Jacobs, K.; Haucke, E.; Navarrete Santos, A.; Grune, T.; Simm, A. Role of advanced glycation end products in cellular signaling. Redox. Biol. 2014, 2, 411-429. [CrossRef]

30. Palanissami, G.; Paul, S.F.D. RAGE and Its Ligands: Molecular Interplay Between Glycation, Inflammation, and Hallmarks of Cancer-a Review. Horm Cancer 2018, 9, 295-325. [CrossRef]

31. Snelson, M.; Coughlan, M.T. Dietary Advanced Glycation End Products: Digestion, Metabolism and Modulation of Gut Microbial Ecology. Nutrients 2019, 11, 215. [CrossRef] 
32. Rapin, J.R.; Wiernsperger, N. Possible links between intestinal permeability and food processing: A potential therapeutic niche for glutamine. Clinics 2010, 65, 635-643. [CrossRef]

33. Shimomoto, T.; Luo, Y.; Ohmori, H.; Chihara, Y.; Fujii, K.; Sasahira, T.; Denda, A.; Kuniyasu, H. Advanced glycation end products (AGE) induce the receptor for AGE in the colonic mucosa of azoxymethane-injected Fischer 344 rats fed with a high-linoleic acid and high-glucose diet. J. Gastroenterol. 2012, 47, 1073-1083. [CrossRef] [PubMed]

34. Xue, J.; Ray, R.; Singer, D.; Bohme, D.; Burz, D.S.; Rai, V.; Hoffmann, R.; Shekhtman, A. The receptor for advanced glycation end products (RAGE) specifically recognizes methylglyoxal-derived AGEs. Biochemistry 2014, 53, 3327-3335. [CrossRef] [PubMed]

35. Xie, J.; Reverdatto, S.; Frolov, A.; Hoffmann, R.; Burz, D.S.; Shekhtman, A. Structural basis for pattern recognition by the receptor for advanced glycation end products (RAGE). J. Biol. Chem. 2008, 283, 27255-27269. [CrossRef]

36. van der Lugt, T.; Venema, K.; van Leeuwen, S.; Vrolijk, M.F.; Opperhuizen, A.; Bast, A. Gastrointestinal digestion of dietary advanced glycation endproducts using an in vitro model of the gastrointestinal tract (TIM-1). Food Funct. 2020, 11, 6297-6307. [CrossRef]

37. Zenker, H.E.; Teodorowicz, M.; Wichers, H.J.; Hettinga, K.A. No Glycation Required: Interference of Casein in AGE Receptor Binding Tests. Foods 2021, 10, 1836. [CrossRef] [PubMed]

38. Hellwig, M.; Bunzel, D.; Huch, M.; Franz, C.M.; Kulling, S.E.; Henle, T. Stability of Individual Maillard Reaction Products in the Presence of the Human Colonic Microbiota. J. Agric. Food Chem. 2015, 63, 6723-6730. [CrossRef] [PubMed]

39. Hellwig, M.; Auerbach, C.; Müller, N.; Samuel, P.; Kammann, S.; Beer, F.; Gunzer, F.; Henle, T. Metabolization of the Advanced

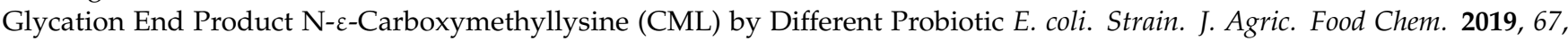
1963-1972. [CrossRef]

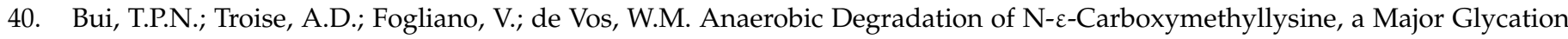
End-Product, by Human Intestinal Bacteria. J. Agric. Food Chem. 2019, 67, 6594-6602. [CrossRef]

41. Fatchiyah, F.; Hardiyanti, F.; Widodo, N. Selective Inhibition on RAGE-binding AGEs Required by Bioactive Peptide Alpha-S2 Case in Protein from Goat Ethawah Breed Milk: Study of Biological Modeling. Acta Inform. Med. 2015, 23, 90-96. [CrossRef]

42. Cordova, R.; Knaze, V.; Viallon, V.; Rust, P.; Schalkwijk, C.G.; Weiderpass, E.; Wagner, K.H.; Mayen-Chacon, A.L.; Aglago, E.K.; Dahm, C.C.; et al. Dietary intake of advanced glycation end products (AGEs) and changes in body weight in European adults. Eur. J. Nutr. 2020, 59, 2893-2904. [CrossRef]

43. Aune, D.; Chan, D.S.; Lau, R.; Vieira, R.; Greenwood, D.C.; Kampman, E.; Norat, T. Dietary fibre, whole grains, and risk of colorectal cancer: Systematic review and dose-response meta-analysis of prospective studies. BMJ 2011, 343, d6617. [CrossRef]

44. Liang, Z.; Chen, X.; Li, L.; Li, B.; Yang, Z. The fate of dietary advanced glycation end products in the body: From oral intake to excretion. Crit. Rev. Food Sci. Nutr. 2020, 60, 3475-3491. [CrossRef] [PubMed]

45. Godfrey, L.; Yamada-Fowler, N.; Smith, J.; Thornalley, P.J.; Rabbani, N. Arginine-directed glycation and decreased HDL plasma concentration and functionality. Nutr. Diabetes 2014, 4, e134. [CrossRef] [PubMed]

46. Thornalley, P.J. Dicarbonyl intermediates in the maillard reaction. Ann. N. Y. Acad. Sci. 2005, 1043, 111-117. [CrossRef]

47. Yeh, W.J.; Hsia, S.M.; Lee, W.H.; Wu, C.H. Polyphenols with antiglycation activity and mechanisms of action: A review of recent findings. J. Food Drug Anal. 2017, 25, 84-92. [CrossRef] [PubMed]

48. Elosta, A.; Ghous, T.; Ahmed, N. Natural products as anti-glycation agents: Possible therapeutic potential for diabetic complications. Curr. Diabetes Rev. 2012, 8, 92-108. [CrossRef]

49. Uribarri, J.; Woodruff, S.; Goodman, S.; Cai, W.; Chen, X.; Pyzik, R.; Yong, A.; Striker, G.E.; Vlassara, H. Advanced glycation end products in foods and a practical guide to their reduction in the diet. J. Am. Diet. Assoc. 2010, 110, 911-916.e12. [CrossRef]

50. Chen, L.; Duan, Z.; Tinker, L.; Sangi-Haghpeykar, H.; Strickler, H.; Ho, G.Y.; Gunter, M.J.; Rohan, T.; Logsdon, C.; White, D.L.; et al. A prospective study of soluble receptor for advanced glycation end-products and colorectal cancer risk in postmenopausal women. Cancer Epidemiol. 2016, 42, 115-123. [CrossRef]

51. Aglago, E.K.; Schalkwijk, C.G.; Freisling, H.; Fedirko, V.; Hughes, D.J.; Jiao, L.; Dahm, C.C.; Olsen, A.; Tjønneland, A.; Katzke, V.; et al. Plasma concentrations of advanced glycation end-products and colorectal cancer risk in the EPIC study. Carcinogenesis 2021, 42, 705-713. [CrossRef]

52. Jiao, L.; Taylor, P.R.; Weinstein, S.J.; Graubard, B.I.; Virtamo, J.; Albanes, D.; Stolzenberg-Solomon, R.Z. Advanced glycation end products, soluble receptor for advanced glycation end products, and risk of colorectal cancer. CancerEpidemiol. Biomark. Prev. 2011, 20, 1430-1438. [CrossRef]

53. de Vos, L.C.; Lefrandt, J.D.; Dullaart, R.P.; Zeebregts, C.J.; Smit, A.J. Advanced glycation end products: An emerging biomarker for adverse outcome in patients with peripheral artery disease. Atherosclerosis 2016, 254, 291-299. [CrossRef] [PubMed]

54. Hellwig, M.; Humpf, H.U.; Hengstler, J.; Mally, A.; Vieths, S.; Henle, T. Quality Criteria for Studies on Dietary Glycation Compounds and Human Health. J. Agric. Food Chem. 2019, 67, 11307-11311. [CrossRef] [PubMed]

55. Chen, G.; Smith, J.S. Determination of advanced glycation endproducts in cooked meat products. Food Chem. 2015, 168, 190-195. [CrossRef] [PubMed] 